\title{
Comparative analysis of two complete Corynebacterium ulcerans genomes and detection of candidate virulence factors
}

Eva Trost ${ }^{1,2}$, Arwa Al-Dilaimi ${ }^{1}$, Panagiotis Papavasiliou ${ }^{1}$, Jessica Schneider ${ }^{1,2,3}$, Prisca Viehoever ${ }^{4}$, Andreas Burkovski ${ }^{5}$, Siomar C Soares ${ }^{6}$, Sintia S Almeida ${ }^{6}$, Fernanda A Dorella ${ }^{6}$, Anderson Miyoshi', Vasco Azevedo ${ }^{6}$, Maria P Schneider ${ }^{7}$, Artur Silva ${ }^{7}$, Cíntia S Santos ${ }^{8}$, Louisy S Santos ${ }^{8}$, Priscila Sabbadini ${ }^{8}$, Alexandre A Dias ${ }^{8}$, Raphael Hirata $\mathrm{Jr}^{8}$, Ana L Mattos-Guaraldi ${ }^{8}$ and Andreas Tauch ${ }^{{ }^{*}}$

\begin{abstract}
Background: Corynebacterium ulcerans has been detected as a commensal in domestic and wild animals that may serve as reservoirs for zoonotic infections. During the last decade, the frequency and severity of human infections associated with C. ulcerans appear to be increasing in various countries. As the knowledge of genes contributing to the virulence of this bacterium was very limited, the complete genome sequences of two $C$. ulcerans strains detected in the metropolitan area of Rio de Janeiro were determined and characterized by comparative genomics: C. ulcerans 809 was initially isolated from an elderly woman with fatal pulmonary infection and C. ulcerans BR-AD22 was recovered from a nasal sample of an asymptomatic dog.

Results: The circular chromosome of C. ulcerans 809 has a total size of 2,502,095 bp and encodes 2,182 predicted proteins, whereas the genome of C. ulcerans BR-AD22 is 104,279 bp larger and comprises 2,338 protein-coding regions. The minor difference in size of the two genomes is mainly caused by additional prophage-like elements in the C. ulcerans BR-AD22 chromosome. Both genomes show a highly similar order of orthologous coding regions; and both strains share a common set of 2,076 genes, demonstrating their very close relationship. A screening for prominent virulence factors revealed the presence of phospholipase D (Pld), neuraminidase $\mathrm{H}(\mathrm{NanH})$, endoglycosidase $E$ (EndoE), and subunits of adhesive pili of the SpaDEF type that are encoded in both C. ulcerans genomes. The rbp gene coding for a putative ribosome-binding protein with striking structural similarity to Shigalike toxins was additionally detected in the genome of the human isolate C. ulcerans 809.

Conclusions: The molecular data deduced from the complete genome sequences provides considerable knowledge of virulence factors in C. ulcerans that is increasingly recognized as an emerging pathogen. This bacterium is apparently equipped with a broad and varying set of virulence factors, including a novel type of a ribosome-binding protein. Whether the respective protein contributes to the severity of human infections (and a fatal outcome) remains to be elucidated by genetic experiments with defined bacterial mutants and host model systems.
\end{abstract}

\section{Background}

Toxigenic Corynebacterium ulcerans was first isolated from a throat lesion of a patient with respiratory diphtheria-like illness in 1926 [1]. These C. ulcerans strains produce a diphtheria toxin, which is similar to

\footnotetext{
* Correspondence: tauch@cebitec.uni-bielefeld.de

${ }^{1}$ Institut für Genomforschung und Systembiologie, Centrum für Biotechnologie, Universität Bielefeld, Universitätsstraße 27, D-33615 Bielefeld, Germany

Full list of author information is available at the end of the article
}

that encoded by toxigenic strains of Corynebacterium diphtheriae [2,3]. This observation has been explained by the fact that $C$. ulcerans may harbor lysogenic $\beta$-corynephages coding for the diphtheria toxin, which is responsible for the systemic symptoms caused by $C$. diphtheriae [4]. Respiratory diphtheria-like illnesses caused by toxigenic $C$. ulcerans strains are increasingly reported from various industrialized countries [5] and became more common than $C$. diphtheriae infections in
C Biomed Central

() 2011 Trost et al; licensee BioMed Central Ltd. This is an Open Access article distributed under the terms of the Creative Commons Attribution License (http://creativecommons.org/licenses/by/2.0), which permits unrestricted use, distribution, and reproduction in any medium, provided the original work is properly cited. 
the United Kingdom [6]. Human infections with toxigenic $C$. ulcerans can be fatal in unvaccinated patients and usually occur in adults, who consumed raw milk $[7,8]$ or had close contact with domestic animals [6]. C. ulcerans has been detected as a commensal not only in domestic animals, but also in wild animals, implying that both groups may serve as reservoirs for the zoonotic transmission of this pathogen $[9,10]$. Several reports demonstrated that toxigenic C. ulcerans strains can be recovered from dairy cows, cats, dogs, goats, pigs, squirrels, free-living otters, dromedary camels, and macaques $[5,11]$. Moreover, C. ulcerans isolates from domestic cats were found to exhibit the predominant ribotypes observed among human clinical isolates, suggesting that strains isolated from cats are a potential reservoir for human infection [12]. Likewise, ribotyping of $C$. ulcerans from a female diphtheria patient and a chronic labial ulcer of her dog revealed that both isolates correspond to a single strain [9]. This example demonstrates that a distinct C. ulcerans strain may infect different hosts.

Beside respiratory diphtheria-like illnesses, C. ulcerans can also cause extrapharyngeal infections in humans, including severe pulmonary infections [13-15]. When $C$. ulcerans isolates from human clinical specimens not fitting reporting criteria for cases of diphtheria were tested for the presence of diphtheria toxin only a portion of strains were positive for the tox gene encoding diphtheria toxin $[16,17]$. These observations indicate that additional factors contribute to the virulence of "non-toxigenic" C. ulcerans strains. A second dermonecrotic toxin with similarity to toxic phospholipase D (Pld) from Corynebacterium pseudotuberculosis appeared to be characteristic of C. ulcerans [18]. The common repertoire of potent toxins in C. diphtheriae, C. ulcerans and C. pseudotuberculosis particularly highlights the close phylogenetic relationship between these three species [19]. Despite this apparent relationship, levels of genomic DNA relatedness and taxonomic analyses of $16 \mathrm{~S}$ rDNA sequences showed that $C$. diphtheriae, $C$. ulcerans and C. pseudotuberculosis are separate taxa within a distinct cluster of the genus Corynebacterium [19]. They can be separated clearly from other pathogenic corynebacteria by chemotaxonomic assays [20]. Hart et al. proposed that the three species evolved from a common ancestor, which parasitized in ungulates in pre-human times [8].

Although C. ulcerans and C. pseudotuberculosis are of increasing medical importance, very little knowledge of their lifestyles and associated virulence factors was available until recently. We extended the genetic knowledge of this corynebacterial cluster by publishing the annotation of four complete genome sequences from C. pseudotuberculosis strains isolated from goat, sheep, cattle, and a rare case of human lymphadenitis [21-23]. In conjunction with the previously evaluated genome sequence from the toxigenic $C$. diphtheriae strain NCTC 13129 [24], a more detailed picture of the closely related corynebacterial pathogens is now available at the genetic and genomic level. In the present study, we established the genome sequences of two C. ulcerans strains (809 and BR-AD22) from human and animal specimens to characterize the architecture of the genome and to compare the predicted gene contents and the repertoires of candidate virulence factors.

C. ulcerans 809 was recovered from a bronchoalveolar lavage (BAL) sample of an 80-year-old woman with rapidly fatal pulmonary infection and a history of chronic bilateral limb ulcers [15]. The woman lived in the metropolitan area of Rio de Janeiro and was hospitalized in coma, with shock and acute respiratory failure. Cultures from the BAL sample revealed the presence of C. ulcerans, and varying antimicrobial therapies were directed to this potential pathogen. This medical treatment resulted in complete healing of the skin lesions. Nevertheless, the cardiorespiratory symptoms of the patient worsened and medical examinations showed evidence of multiple organ failures. The patient died 23 days after hospitalization. Subsequent toxigenicity tests and PCR assays evaluating the production of diphtheria toxin by C. ulcerans 809 were ambiguous and it was concluded that the unusual nature of the pathogen possibly contributed to the patient's death [15].

The second isolate investigated in this study, C. ulcerans BR-AD22, was obtained from a nasal sample of a 5year-old female dog kept in an animal shelter in the metropolitan area of Rio de Janeiro [25]. General clinical aspects and laboratory findings revealed this dog as an asymptomatic carrier of C. ulcerans. Toxigenicity assays showed the presence of phospholipase $\mathrm{D}$ in C. ulcerans BR-AD22, but were negative for the presence of diphtheria toxin [25]. In the following sections, we present the results of the genome sequencing project and the comparative analysis of the genomes from the selected $C$. ulcerans strains, thereby focusing on the relevant differences in the gene content and the repertoires of virulence factors.

\section{Results and discussion}

\section{General features and architecture of the $C$. ulcerans genomes}

The genome sequences of C. ulcerans 809 from a human clinical source and C. ulcerans BR-AD22 from an asymptomatic dog were determined by pyrosequencing using a quarter of a sequencing run with the Genome Sequencer FLX Instrument for each strain. The resulting reads were assembled with the Newbler Assembler software, and the remaining gaps were closed by PCR strategies that were supported by the related reference contig arrangement 


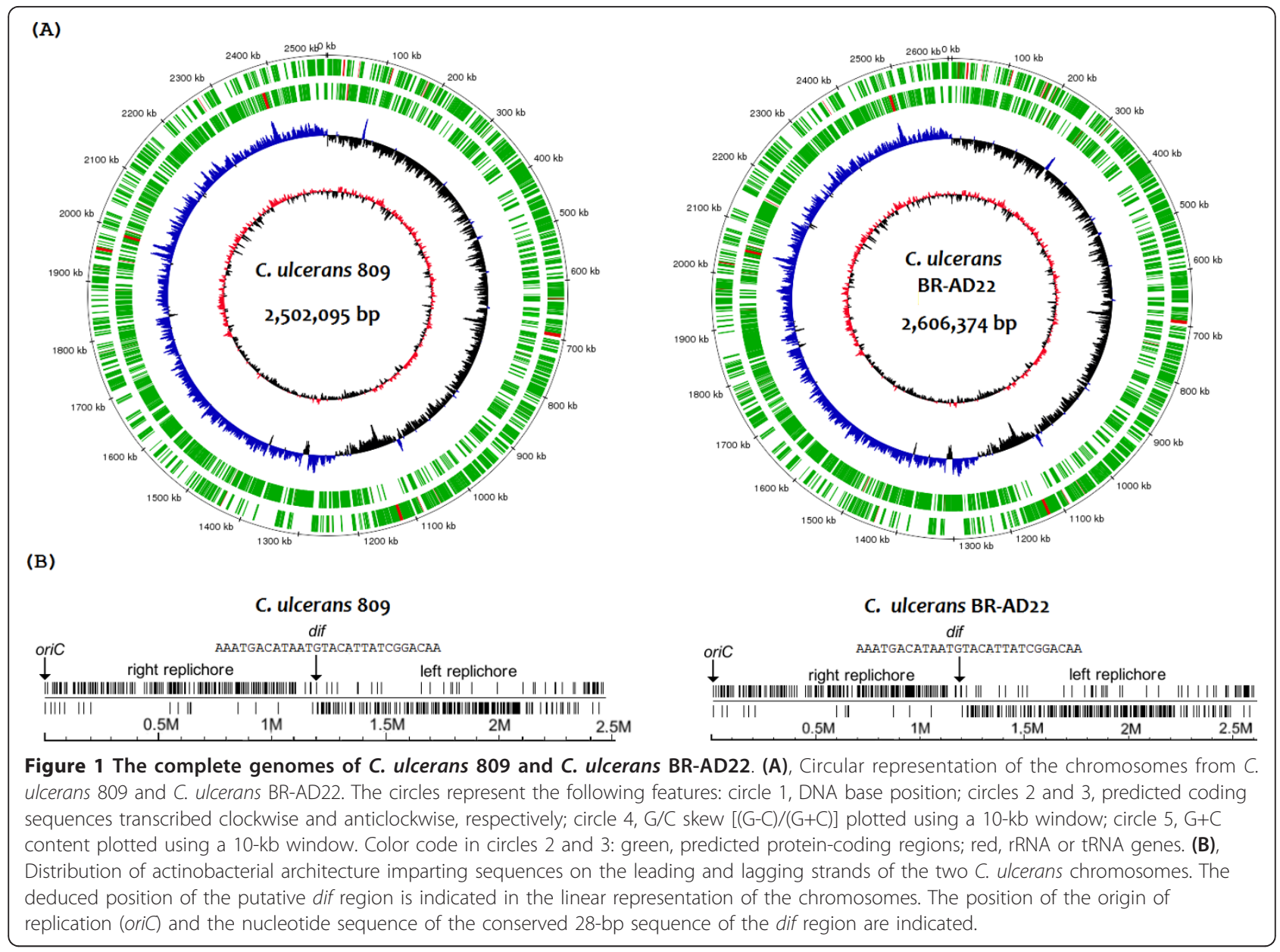

tool r2cat [26] using the genome sequence of C. pseudotuberculosis FRC41 as a reference [21]. The final assemblies of the two genomic DNA sequences yielded circular chromosomes with a mean $\mathrm{G}+\mathrm{C}$ content of $53 \%$ (Figure 1A), which is very similar to that of the closely related species C. pseudotuberculosis (52.2\%) [21] and C. diphtheriae (53.5\%) [24]. The chromosome of C. ulcerans 809 has a size of 2,502,095 bp and is thus 104,279 bp smaller than that of C. ulcerans BR-AD22 (Table 1). The subsequent gene prediction and annotation of the genome sequences was performed automatically with the GenDB system [27]. After manual curation of the annotation, 2,182 proteincoding regions were detected in the $C$. ulcerans 809 genome, whereas 2,338 protein-coding regions were predicted in the genome sequence of $C$. ulcerans BR-AD22. These data already indicated strain-specific differences in the gene repertoires of both C. ulcerans isolates. Relevant data and general features deduced from the genome sequences of C. ulcerans 809 and C. ulcerans BR-AD22 are summarized in Table 1.

The calculation of the $\mathrm{G} / \mathrm{C}$ skew $[(\mathrm{G}-\mathrm{C}) /(\mathrm{G}+\mathrm{C})]$ of both genome sequences revealed a bi-directional replication mechanism for the $C$. ulcerans chromosome (Figure 1A). According to the presence of conserved DnaA boxes [TTATC $(\mathrm{C} / \mathrm{A}) \mathrm{A}(\mathrm{C} / \mathrm{A}) \mathrm{A}$ ], the origin of chromosomal replication (oriC) is located downstream of the $d n a A$ gene and has a computed length of $423 \mathrm{bp}$

Table 1 General features of the genome sequences of $C$. ulcerans 809 and C. ulcerans BR-AD22

\begin{tabular}{lll}
\hline Feature & C. ulcerans 809 & C. ulcerans BR-AD22 \\
\hline Genome size (bp) & $2,502,095$ & $2,606,374$ \\
Sequenced bases & $106,993,163$ & $59,757,327$ \\
Genome coverage & $42.8 \times$ & $22.9 \times$ \\
G+C content (\%) & 53.3 & 53.4 \\
Coding sequences & 2,182 & 2,338 \\
Coding density (\%) & 87.7 & 87.8 \\
Average gene length (bp) & 1,006 & 979 \\
ribosomal RNAs & $4 \times(16 S-23 S-5 S)$ & $4 \times(16 S-23 S-5 S)$ \\
transfer RNAs & 52 & 52 \\
Prophages $_{\text {CRISPRs }}{ }^{a}$ & 1 & 4 \\
\hline
\end{tabular}

\footnotetext{
a Abbreviation: Clustered Regularly Interspaced Short Palindromic Repeats
} 
[28]. The plot of the G/C skew additionally indicated the presence of a putative dif region involved in replication termination [29] at the expected position of about $180^{\circ}$ from oriC, dividing the chromosome of C. ulcerans in two replichores of similar size (Figure 1A). For a more precise detection of the dif region, the distribution of the actinobacterial architecture imparting sequences G(A/T/C)GGGGGA and (T/C)GGGGGAG [30] was plotted on the leading and lagging strands of the $C$. ulcerans chromosomes (Figure 1B). Both linear plots show a characteristic distribution of the architecture imparting sequences, as these octamers are overrepresented on the leading strands and underrepresented on the lagging strands. Putative dif regions were detected at around 1,193 $\mathrm{kb}$ of the chromosomal map of C. ulcerans 809 and at 1,195 kb of the C. ulcerans BR-AD22 chromosome (Figure 1B). In accordance with this computation, the respective DNA regions of the C. ulcerans chromosomes contain a conserved 28-bp sequence that shows similarity to the consensus sequence of actinobacterial dif sites [29]. Moreover, four $r r n$ operons were identified in the genome sequences of C. ulcerans 809 and C. ulcerans BR-AD22. All rrn operons are located on the leading strands of the C. ulcerans chromosome; two are present on the right and two on the left replichore, respectively. In summary, the structural analysis of the complete genome sequences of two C. ulcerans strains revealed the typical architecture of a corynebacterial chromosome in this species [31,32] with the presence of strain-specific variations that were investigated in more detail by the following comparative analysis.

\section{Comparative analysis of the gene order in the C. ulcerans genomes}

A synteny analysis was performed by plotting reciprocal best BLASTP matches [33] to compare the gene order in the chromosomes of C. ulcerans 809 and C. ulcerans BR-AD22. This computation revealed a highly conserved order of orthologous genes between the two C. ulcerans chromosomes, as only three breakpoints of synteny were detectable in the left replichore (Figure 2A). These breakpoints are clearly indicative of the insertion of additional DNA regions into the chromosome of $C$. ulcerans BR-AD22. The annotation of the respective gene regions suggests that the breakpoints are due to the presence of prophage-like elements in the $C$. ulcerans BR-AD22 genome, named ФCULC22II, ФCULC22III, and ФCULC22IV (Figure 2A). An additional prophage-like region (ФCULC809I and ФCULC22I) is present at identical positions in both C. ulcerans chromosomes (Figure 2A). Considering a total size of 99.9 $\mathrm{kb}$ for the three additional prophage-like elements in $C$. ulcerans BR-AD22 (Table 2), the difference in size of the two C. ulcerans genomes is mainly caused by variations in the individual repertoire of prophages. This result indicates that the sequenced $C$. ulcerans strains are very closely related, although they were originally isolated at different locations from a human clinical specimen and the nares of an asymptomatic dog.

The gene order in C. ulcerans 809 was moreover compared to those in the taxonomically closely related species C. diphtheriae and C. pseudotuberculosis. This comparison revealed also a highly conserved order of orthologous
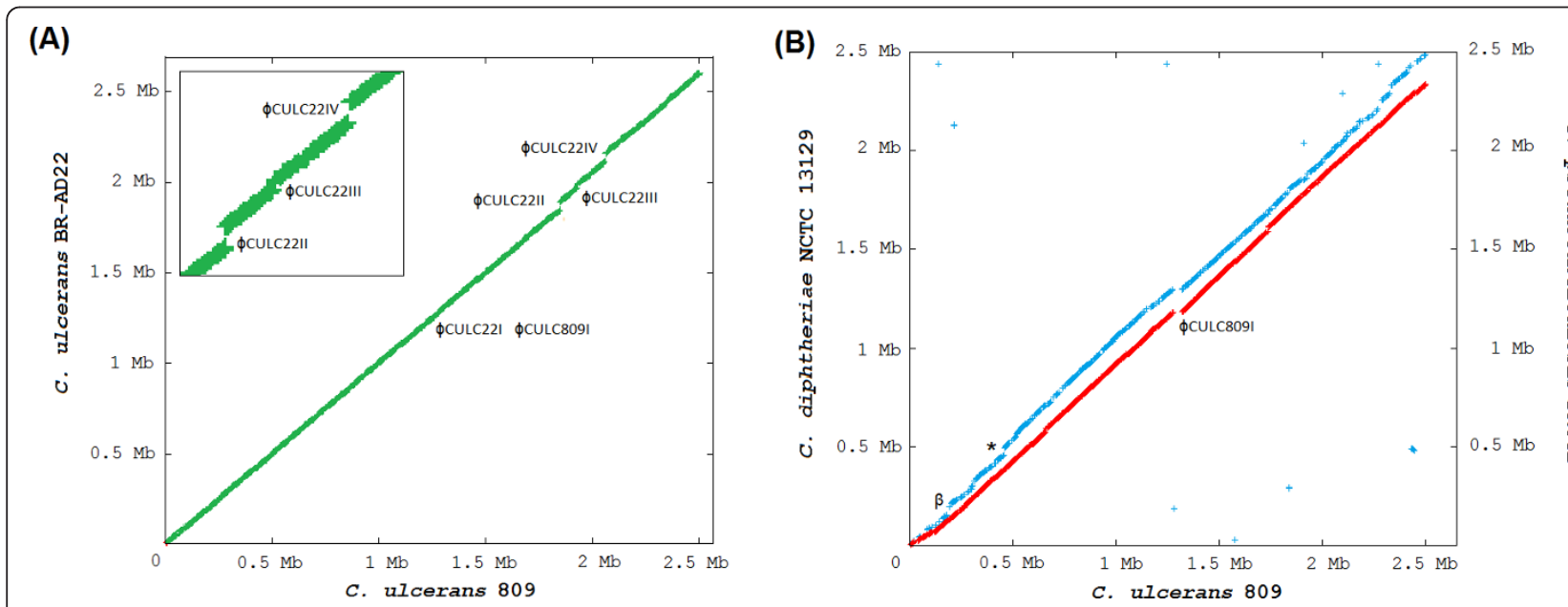

Figure 2 Comparative analysis of the gene order in C. ulcerans, C. diphtheriae and C. pseudotuberculosis. (A), Synteny between the sequenced chromosomes of C. ulcerans 809 and C. ulcerans BR-AD22. (B), Synteny between the chromosome of C. ulcerans 809 and those from C. diphtheriae NCTC 13129 (blue) and C. pseudotuberculosis FRC41 (red). The graphs represent X-Y plots of dots forming syntenic regions between the selected chromosomes. Each dot represents a predicted protein having an orthologous counterpart in another corynebacterial genome, with co-ordinates corresponding to the position of the respective coding region in each genome. Orthologous proteins were detected by reciprocal best BLASTP matches. The genomic positions of putative prophages detected in $C$. ulcerans are marked in the synteny plots. Symbols: $\beta, \beta-$ corynephage of C. diphtheriae NCTC 13129; asterisk, nitrate reductase gene region of C. diphtheriae NCTC 13129. 
genes in the genomes of C. diphtheriae NCTC 13129 and C. pseudotuberculosis FRC41 (Figure 2B), which is consistent with the previous observation that genetic rearrangements are rare in genomes of species belonging to the main lineage of the genus Corynebacterium [32]. Only one remarkable breakpoint of synteny was observed when comparing the order of genes in C. ulcerans 809 and $C$. pseudotuberculosis FRC41. This breakpoint is located at 1,300 kb of the chromosomal map of C. ulcerans 809 and is caused by the integration of the prophage-like element ФCULC809I (Figure 2B).

The synteny analysis between $C$. ulcerans 809 and $C$. diphtheriae NCTC 13129 revealed two additional breakpoints within the highly conserved order of genes. The first breakpoint is located about $154 \mathrm{~kb}$ downstream of ori $C$ and comprises the genes of the corynephage $\beta$ (DIP0180 to DIP0222), among others coding for the diphtheria toxin in C. diphtheriae NCTC 13129. The lysogenic $\beta$-corynephage is completely missing in the genome of C. ulcerans 809 . The second breakpoint of synteny is located at about $457 \mathrm{~kb}$ and comprises genes for nitrate reductase and associated protein-coding regions for the synthesis of the molybdenum cofactor (DIP0492 to DIP0507) [24]. This observation is in line with taxonomic reports using nitrate reductase activity as a distinct metabolic marker to distinguish between $C$. diphtheriae (gravis, intermedius, mitis) and C. ulcerans isolates, as nitrate reductase activity was not detectable in the latter species [20].

\section{Comparative analysis of prophage-like sequences in the C. ulcerans genomes}

According to the synteny analysis, the variation in the repertoire of prophage-like regions is a remarkable difference between the sequenced $C$. ulcerans genomes, as one putative prophage was identified in the C. ulcerans 809 genome and four prophage-like regions were detected in the genome sequence of C. ulcerans BRAD22 (Table 2). Accordingly, this genome project provides the first molecular genetic data about corynephages infecting the species $C$. ulcerans that may also harbor $\beta$-corynephages coding for the diphtheria toxin [34]. The respective genomic regions of C. ulcerans 809 and C. ulcerans BR-AD22 were characterized in more detail and the deduced genetic maps of the putative prophages are presented in Figure 3.

The prophage-like regions $\Phi$ CULC22I from C. ulcerans BR-AD22 and ФCULC809I from C. ulcerans 809 have a size of about $42 \mathrm{~kb}$ and are characterized by highly similar genetic maps. Both prophage-like elements were detected at the same genomic position and apparently integrated at slightly different sites into a coding region for a hypothetical protein (CULC22_01157 and CULC809_01141) that may represent the integration site of these phages in the $C$. ulcerans chromosome. Minor differences between the putative prophages were detected in the number of predicted genes: the ФCULC22I region comprises 42 genes (CULC22_01158 to CULC22_01199), whereas 45 genes were assigned to the $\Phi$ CULC809I region (CULC809_01142 to CULC809_01186). According to BLASTP matches and global amino acid sequence alignments, both putative prophages share 36 genes that code for gene products with a least $98 \%$ amino acid sequence identity. These values clearly demonstrate the very close relationship of both prophages from different C. ulcerans strains.

The prophage-like region $\Phi$ CULC22II from C. ulcerans BR-AD22 has a size of $44.9 \mathrm{~kb}$ and comprises 60 genes (Figure 3). A sequence comparison with the corresponding region in the genome of C. ulcerans 809 revealed that this putative prophage is apparently integrated into the ortholog of CULC809_01647 encoding a hypothetical protein. The integration of the putative phage into the chromosome of C. ulcerans BR-AD22 probably divided this gene into two pseudogenes (CULC22_01663 and CULC22_01724) that are located directly adjacent to the $\Phi C U L C 22 \mathrm{II}$ region. This view is supported by the presence of 8 -bp direct repeats that are located at the borders of this prophage-like element and may represent the integration site of ФCULC22II in C. ulcerans (Figure 3).

The third prophage-like region of $C$. ulcerans BRAD22 (ФCULC22III) is located adjacent to a second tRNA $^{\text {Lys }}$ gene that may represent the integration site due to the presence of a 21-bp direct repeat, which is

Table 2 General features of prophage-like elements detected in the $C$. ulcerans genomes

\begin{tabular}{|c|c|c|c|c|c|c|}
\hline Name & Size & $\begin{array}{l}\mathrm{G}+\mathrm{C} \\
\text { content }\end{array}$ & $\begin{array}{l}\text { No. of } \\
\text { CDS }\end{array}$ & $\begin{array}{l}\text { CDS with assigned } \\
\text { function }\end{array}$ & Integration site & Attachment site \\
\hline ФCULC8091 & $\begin{array}{l}41.4 \\
k b\end{array}$ & $53 \%$ & 45 & 17 & CULC809_01141 & Not detected \\
\hline ФCULC22I & $42 \mathrm{~kb}$ & $53 \%$ & 42 & 13 & CULC22_01157 & Not detected \\
\hline ФCULC22II & $\begin{array}{l}44.9 \\
\mathrm{~kb}\end{array}$ & $55 \%$ & 60 & 18 & $\begin{array}{l}\text { Between CULC22_01663 and } \\
\text { CULC22_01724 }\end{array}$ & TTAGATAC \\
\hline$\Phi C U L C 22 I I \mid$ & $14 \mathrm{~kb}$ & $57 \%$ & 19 & 9 & tRNA ${ }^{\text {Lys }}$ & TTCAAGTCCCTGATGGCGCAC \\
\hline ФCULC22IV & $41 \mathrm{~kb}$ & $54 \%$ & 53 & 16 & tRNA $^{\text {Thr }}$ & TTGAGCTGGAGATGGGACTTGAACCC \\
\hline
\end{tabular}




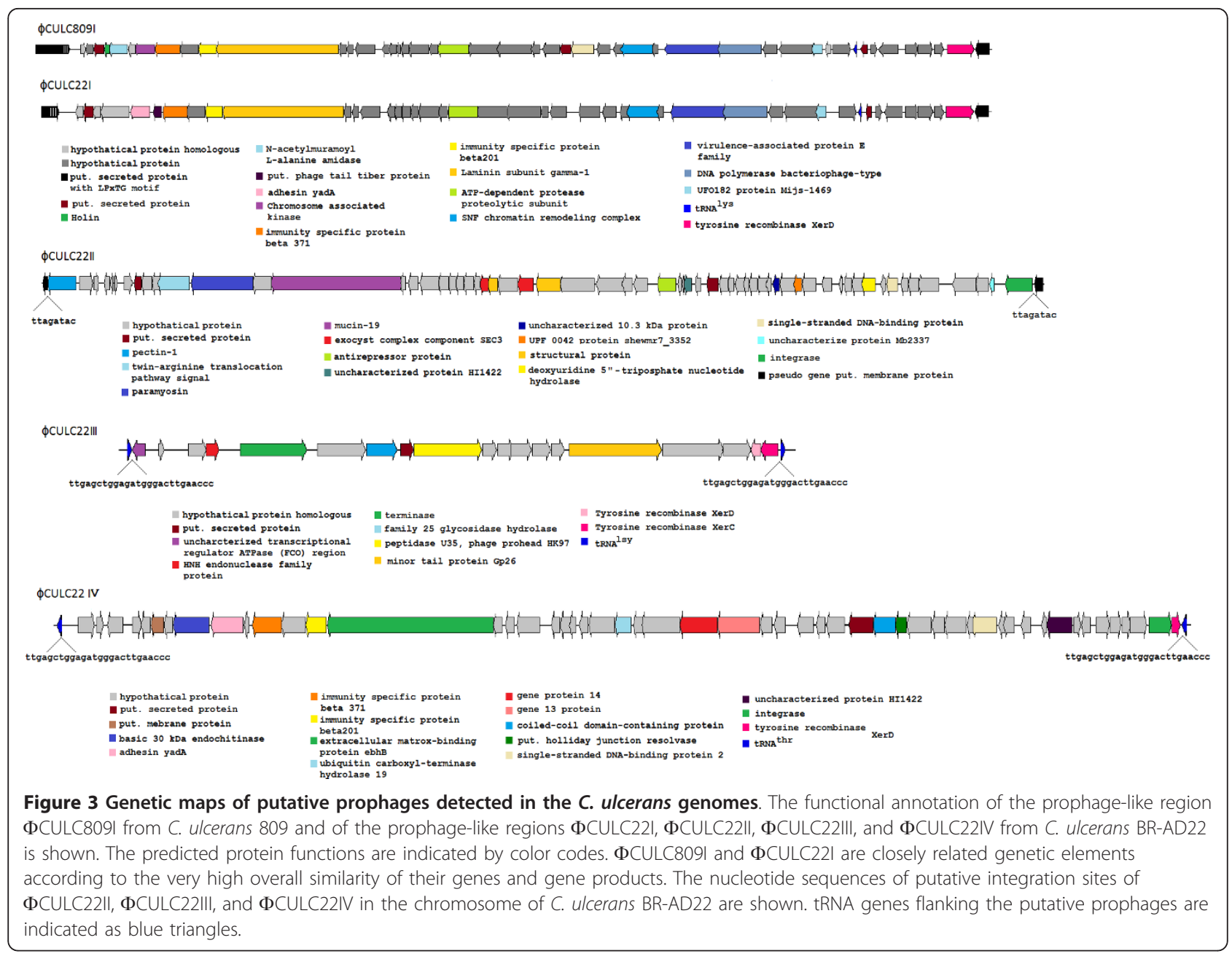

part of this tRNA ${ }^{\text {Lys }}$ gene and flanks the prophage-like region (Figure 3). The ФCULC22III region has a size of about $14 \mathrm{~kb}$ and comprises 19 genes (CULC22_01793 to CULC22_01811). The size difference between the prophage-like regions of $C$. ulcerans BR-AD22 suggests that at least $\Phi C U L C 22 I I I$ is incomplete and a defective remnant of a formerly active corynephage (Table 2 ). The prophage-like region $\Phi$ CULC22IV of C. ulcerans BR-AD22 comprises 53 genes (CULC22_01925 to CULC22_01977), has a size of about $41 \mathrm{~kb}$ and is located adjacent to a tRNA ${ }^{\text {Thr }}$ gene. A 26-bp direct repeat, which is part of the tRNA gene, may represent the integration site of the respective corynephage.

The previous experimental characterization of $C$. ulcerans 809 suggested the presence of a $\beta$-corynephage in this strain, as PCR assays amplified putative fragments of the tox gene coding for the diphtheria toxin [15]. However, neither the tox gene nor other DNA segments of the $\beta$-corynephage were identified in the genome sequences of C. ulcerans 809 and C. ulcerans BR-AD22. This result is also obvious when considering the lack of synteny between the chromosomes of $C$. diphtheriae NCTC 13129 and C. ulcerans 809 at the integration site of the corynephage $\beta$ (Figure 2B). Nevertheless, remnants of a putative corynephage are located adjacent to the tRNA $^{\text {Arg }}$ gene, which comprises the attachment site for the $\beta$-phage [35]. The genes CULC809_00176 and CULC22_00173 code for putative phage-type integrases in the genome sequence of $C$. ulcerans BR-AD22 and $C$. ulcerans 809 . The respective tyrosine recombinase from C. ulcerans shares $92 \%$ amino acid sequence identity with the integrase of the $\beta$-corynephage integrated in the genome of C. diphtheriae NCTC 13129 and is encoded adjacent to the tRNA ${ }^{\text {Arg }}$ gene. This gene annotation supports the assumption that a lysogenic $\beta$-corynephage-like phage had been integrated in both $C$. ulcerans genomes in former times.

\section{Detection and comparative analysis of CRISPR regions in} the $C$. ulcerans genomes

A screening of the genome sequences of C. ulcerans 809 and C. ulcerans BR-AD22 with the CRISPRFinder 
program [36] revealed the presence of three loci of socalled clustered regularly interspaced short palindromic repeats (CRISPRs) (Table 3 ). These repeat regions are often associated with CRISPR-associated (cas) genes and may provide acquired immunity against bacteriophages and other foreign genetic elements by means of a sequence specificity that is determined by similarities between the spacer sequences and foreign DNA [37]. CRISPR locus I is present in both sequenced C. ulcerans genomes and flanked by four cas genes (CULC809_00031 to CULC809_00034 and CULC22_00029 to CULC22_00032). The direct repeats of this locus are $29 \mathrm{bp}$ in length and separated by spacers with variable nucleotide sequences that are completely different in both $C$. ulcerans strains. The number of CRISPR spacers is also different in both strains, whereas the cas genes and the consensus sequences of the CRISPRs are identical (Table 3).

Similar structural features were observed for the second array of CRISPRs in the two C. ulcerans genomes (Table 3). CRISPR locus II is flanked by six cas genes (CULC809_00109 to CULC809_00114 and CULC22_00106 to CULC22_00111). The consensus sequence of this CRISPR has a length of $36 \mathrm{bp}$, separated by either 12 spacers in C. ulcerans 809 or 10 spacers in C. ulcerans BR-AD22. The spacers present in C. ulcerans 809 are different again to those located in the corresponding locus of the C. ulcerans BR-AD22 genome. In contrast to CRISPR loci I and II, a third putative CRISPR region in C. ulcerans 809 and C. ulcerans BR-AD22 is not specified by the presence of cas genes in the direct proximity. The CRISPR of this genomic region has a length of $29 \mathrm{bp}$, and the number of spacer sequences revealed the largest variation between both $C$. ulcerans strains, with 67 spacers present in $C$. ulcerans 809 and 32 spacers in the genome of C. ulcerans BR-AD22 (Table 3). The detection of CRISPRs in the genome of $C$. ulcerans and the sequence variations of the CRISPR loci suggests the use of these molecular genetic markers for a more precise and high-resolution typing of closely related $C$. ulcerans strains from clinical specimens and animal reservoirs. A macroarray-based hybridization method, named spacer oligonucleotide typing ("spoligotyping"), has already been developed to study the polymorphism of spacer sequences in CRISPR loci of distinct ribotypes from epidemic $C$. diphtheriae isolates [38].

\section{Comparative analysis of the predicted gene content of the $C$. ulcerans genomes}

The initial analyses of genomic features and the genome architecture of C. ulcerans 809 and C. ulcerans BRAD22 revealed considerable similarities between the sequenced genomes. The very close relationship of both strains is also evident when calculating the common gene repertoire by reciprocal best BLASTP matches with the EDGAR software [33]. Both $C$. ulcerans strains share a common set of 2,076 genes and are therefore characterized by small numbers of strain-specific genes, named singletons in this study [33]. As most strain-specific genes of the animal isolate C. ulcerans BR-AD22 were assigned to the additional prophage-like regions ФCULC22II, ФCULC22III and ФCULC22IV, only 92 coding regions of this strain were finally regarded as singletons sensu stricto, of which 13 genes were annotated with putative physiological functions (Table 4). This group of singletons includes four genes coding for typical two-component signal transduction systems consisting of a sensor histidine kinase and a DNA-binding response regulator. One two-component system is encoded by the genes CULC22_00235 and CULC22_ 00236 that are located downstream of CULC22 00237 coding for a putative glycerol-3-phosphate transporter (Figure 4A). The genes of the second two-component system (CULC22_00055 and CULC22_00056) are located adjacent to a remnant of a transposase gene (CULC22_00054), suggesting a former event of horizontal gene transfer in this genomic region (Figure $4 \mathrm{~A}$ ). Other singletons of C. ulcerans BR-AD22 encode putative enzymes with unknown specificities, such as CULC22_02221 (SGNH-hydrolase family protein) and CULC22_02229 (esterase-lipase family protein). Two genes encoding surface-anchored proteins with LPxTG motif, including the $s p a D$ gene for the major pilin subunit of an adhesive pilus structure, were also detected as singletons (Table 4). The SpaD protein of C. ulcerans BR-AD22 differs in its amino acid sequence when compared with the functional counterpart CULC809_01952

Table 3 Structural features of CRISPR loci detected in the C. ulcerans genomes

\begin{tabular}{lllll}
\hline Name & No. of cas genes & No. of spacers & CRISPR size & CRISPR consensus sequence in both genomes \\
\hline CRISPR809_I & 4 & 28 & $29 \mathrm{bp}$ & CTTTTCTCCGCGTACGCGGAGGTAGTTCC \\
CRISPR22_I & 4 & 38 & $29 \mathrm{bp}$ & ACCTCAATGAAAGGCTGCGACCGAAGCCGCAGCGAC \\
\hline CRISPR809_II & 6 & 12 & $36 \mathrm{bp}$ & $36 \mathrm{bp}$ \\
CRISPR22_II & 6 & 10 & $29 \mathrm{bp}$ & CTTTTCTCCGCGTATGCGGAGGTAGTTCC \\
\hline CRISPR809_III & 0 & 67 & $29 \mathrm{bp}$ & \\
\hline
\end{tabular}


Table 4 Deduced functions of strain-specific genes in C. ulcerans 809 and C. ulcerans BR-AD22

\begin{tabular}{|c|c|c|c|}
\hline Identifier & Gene & $\mathrm{G}+\mathrm{C}$ content & Proposed function of deduced protein \\
\hline CULC809_00055 & nreB & $51.2 \%$ & Sensor histidine kinase (two-component system) \\
\hline CULC809_00056 & nreC & $48.0 \%$ & Response regulator (two-component system) \\
\hline CULC809_00086 & $\operatorname{tcs} 55$ & $57.1 \%$ & Sensor histidine kinase (two-component system) \\
\hline CULC809_00087 & $\operatorname{tcs} R 5$ & $51.1 \%$ & Response regulator (two-component system) \\
\hline CULC809_00176 & intC & $53.1 \%$ & Phage-related integrase \\
\hline CULC809_00177 & $r b p$ & $45.1 \%$ & Putative ribosome binding protein \\
\hline CULC809_01822 & - & $45.3 \%$ & Surface-anchored protein \\
\hline CULC809_01940 & - & $52.9 \%$ & Surface-anchored protein \\
\hline CULC809_01952 & spaD & $46.7 \%$ & Surface-anchored protein (fimbrial subunit) \\
\hline CULC809_01964 & vsp2 & $49.3 \%$ & Venome serine protease \\
\hline CULC22_00055 & tCsR5 & $50.8 \%$ & Response regulator (two-component system) \\
\hline CULC22_00056 & $\operatorname{tcs} 55$ & $48.1 \%$ & Sensor histidine kinase (two-component system) \\
\hline CULC22_00171 & - & $46.8 \%$ & DNA-binding transcriptional regulator \\
\hline CULC22_00173 & intC & $48.7 \%$ & Phage-related integrase \\
\hline CULC22_00174 & - & $51.1 \%$ & Helix-turn-helix domain protein \\
\hline CULC22_00235 & tCsR1 & $50.3 \%$ & Response regulator (two-component system) \\
\hline CULC22_00236 & $\operatorname{tcs} 1$ & $52.9 \%$ & Sensor histidine kinase (two-component system) \\
\hline CULC22_01271 & tetR4 & $52.7 \%$ & TetR-family transcriptional regulator \\
\hline CULC22_02106 & spaD & $45.1 \%$ & Surface-anchored protein (fimbrial subunit) \\
\hline CULC22_02199 & - & $48.6 \%$ & Surface-anchored protein \\
\hline CULC22_02221 & - & $49.0 \%$ & SGNH-hydrolase family protein \\
\hline CULC22_02229 & - & $61.3 \%$ & Esterase-lipase family protein \\
\hline CULC22_02230 & - & $62.3 \%$ & DNA-binding transcriptional regulator \\
\hline
\end{tabular}

\section{(A)}
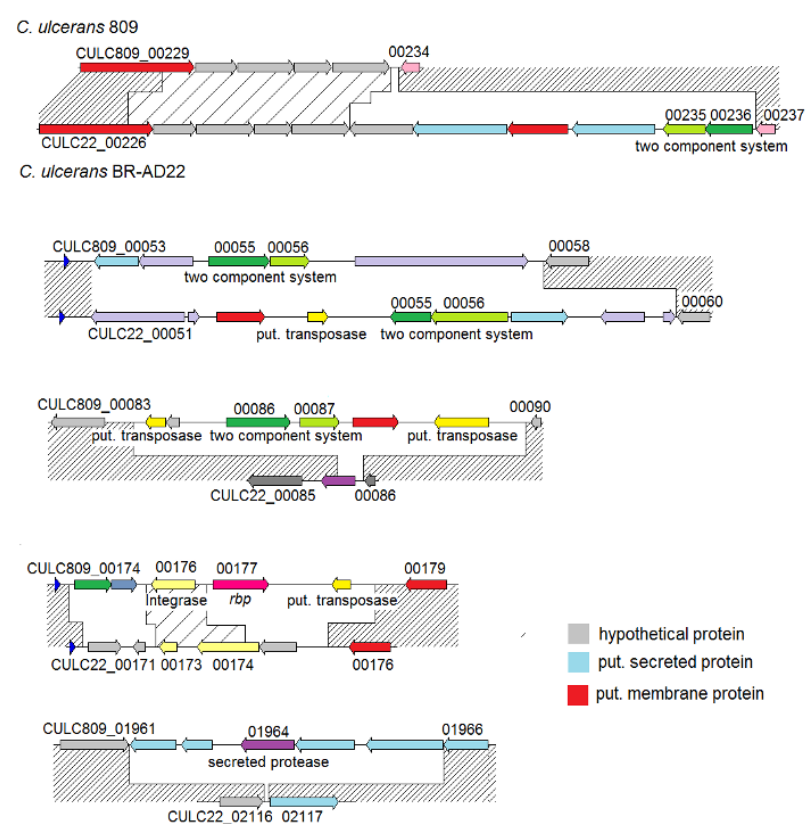

(B)

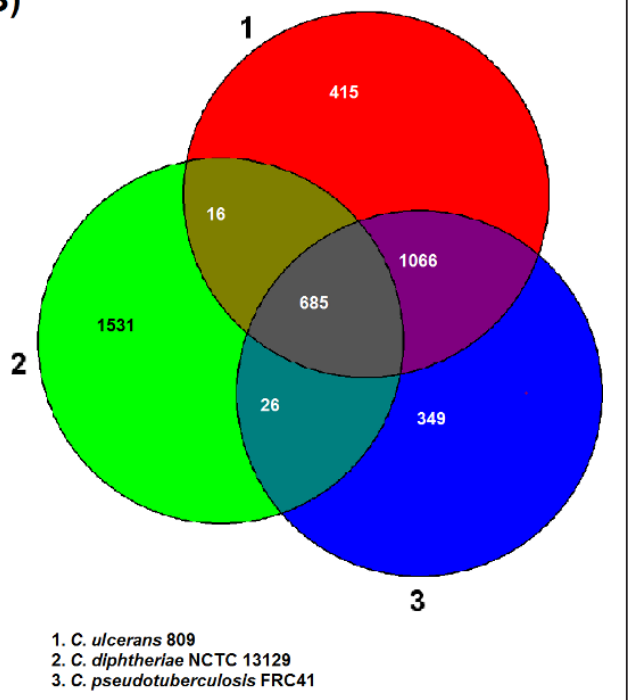

Figure 4 Intra- and inter-species comparison of the predicted gene content of the $\boldsymbol{C}$. ulcerans genomes. (A), Selected examples of genomic regions comprising strain-specific genes in C. ulcerans. Orthologous gene regions are shaded gray. (B), Venn diagram comparing the gene content of C. ulcerans 809, C. diphtheriae NCTC 13129 and C. pseudotuberculosis FRC41. The Venn diagram shows the number of shared and species-specific genes among the three corynebacterial genomes. 
from $C$. ulcerans 809 , demonstrating that the adhesive pili of the two $C$. ulcerans strains vary significantly in the primary sequence of their major pilins that in principle constitute the shaft of the corynebacterial pilus structure [39].

Furthermore, the search for singletons by reciprocal best BLASTP matches with EDGAR revealed 90 strainspecific genes for the human isolate C. ulcerans 809, of which 10 were annotated with putative functions (Table $4)$. The genome annotation revealed two gene pairs coding for two-component systems as singletons, including CULC809_00086 and CULC_00087 that are flanked by transposase genes (Figure 4A). The vsp2 gene (CULC809_01964) coding for a secreted serine protease and the rbp gene (CULC809_00177) encoding a putative ribosome-binding protein were also recognized as singletons in the genome of $C$. ulcerans 809 . Both gene products represent candidate virulence factors of $C$. ulcerans 809 (see below). Interestingly, the rbp gene is located between a gene coding for a putative phage integrase (CULC809_00176) and a transposase gene (CULC809_00178) and is moreover specified by the low $\mathrm{G}+\mathrm{C}$ content of $45.1 \%$ (Table 4 ), suggesting the horizontal transfer of this gene to C. ulcerans 809. In summary, the detection and functional assignments of singletons indicates that the repertoire of potential virulence factors of the sequenced C. ulcerans strains is different in the two selected isolates from a human and animal source, respectively.

\section{Inter-species comparison of the gene content detected in} the $C$. ulcerans genomes

The gene content of C. ulcerans 809 was compared in respect to encoded proteins with those of C. pseudotuberculosis FRC41 and C. diphtheriae NCTC 13129. This comparative content analysis showed that 685 predicted proteins of C. ulcerans 809 (31.4\% of the total number of predicted proteins) share homologs in the genomes of the closely related species (Figure 4B). The number of core genes is remarkably low, as a similar calculation with genomic data from pathogenic and non-pathogenic corynebacteria, including $C$. diphtheriae, Corynebacterium jeikeium, Corynebacterium efficiens, and Corynebacterium glutamicum, revealed 835 genes as a conserved corynebacterial backbone [40]. These numbers indicate a larger variation in the deduced gene repertoires from C. ulcerans, C. pseudotuberculosis and C. diphtheriae than initially expected when considering their close phylogenetic relationship. Nevertheless, the conserved genetic backbone detected in both comparative studies comprises genes for the basic cellular machineries, such as the components involved in DNA replication, DNA repair, transcription, and protein biosynthesis, the conserved corynebacterial regulatory systems [41], the components of the central carbon and energy metabolism, and of biosynthesis routes for amino acids, cofactors, purines, and pyrimidines, as well as the machinery involved in cell wall formation $[40,42]$.

Interestingly, C. ulcerans and $C$. diphtheriae share only 16 genes, which are not present in the genome of C. pseudotuberculosis (Figure 4B). According to the genome annotations, most genes of this group encode putative transporters and secreted proteins with unknown functions. On the other hand, C. ulcerans and C. pseudotuberculosis share 1066 homologous genes (Figure $4 \mathrm{~B})$, indicating that these species are more closely related to each other than to $C$. diphtheriae. The physiological functions encoded by this set of genes may also reflect differences in the lifestyle of the animal pathogens and the human pathogen $C$. diphtheriae. The group of homologous coding regions of C. ulcerans and C. pseudotuberculosis includes 733 genes with unknown function. However, the functional analysis of the remaining genes revealed that they mainly code for transport systems, including a variety of permeases and ABC-type transporters for the uptake of metal ions, indicating an adaptation of both species to the availability of trace elements in their ecological niche. Moreover, genes encoding the subunits of urease and accessory proteins of urease were only detected in the genomes of C. ulcerans and C. pseudotuberculosis, which is consistent with previous data describing these species as urease-positive, whereas $C$. diphtheriae was tested negative for urease activity $[20,43]$.

As expected, the pld gene encoding the sphingomyelin-degrading phospholipase D was found among the subset of genes homologous for C. ulcerans and C. pseudotuberculosis $[18,43]$. Phospholipase D represents the major virulence factor of C. ulcerans and C. pseudotuberculosis and facilitates the persistence and spread of these bacteria within the mammalian hosts $[44,45]$. The cpp gene encoding the "corynebacterial protease CP40" was also detected in the genome of $C$. ulcerans. This secreted protein was described previously as a protective antigen of C. pseudotuberculosis [46,47]. As the comparison of the gene content among the three species of the C. diphtheriae cluster revealed notable differences in the repertoire of virulence factors, the genome sequences of C. ulcerans 809 and C. ulcerans BR-AD22 were searched for the presence of additional genes probably contributing to the virulence of these strains.

\section{Detection of genes encoding candidate virulence factors in C. ulcerans 809 and C. ulcerans BR-AD22}

To extend the view on proteins probably contributing to the virulence of C. ulcerans 809 and C. ulcerans BRAD22, the annotated proteomes of both strains were screened for protein precursors with N-terminal 
secretion signals [48] and proteins containing a C-terminal LPxTG motif allowing their anchoring to the bacterial cell wall [49]. This bioinformatic search revealed twelve candidate virulence factors that are common to both strains and two additional proteins encoded only in the genome of C. ulcerans 809 (Table 5). In addition to the tspA and $v s p 1$ gene products representing secreted proteins of the serine protease type in both strains, the $v s p 2$ gene (CULC809_01964) encodes an additional extracellular serine protease in C. ulcerans 809. This enzyme family can show a wide range of pathogenic potentials when interacting with tissue components of the host or with components of the host's defense system [50]. The redundancy of the corresponding enzymatic activities in C. ulcerans 809 might promote the interaction of the pathogen with the host and the survival of the bacterium in an unfavorable environment.

Another potential virulence factor of C. ulcerans is the extracellular neuraminidase NanH (Table 5). Some enzymatic properties of this enzyme were characterized in a previous study indicating that this thermo-labile protein has a temperature optimum of $37^{\circ} \mathrm{C}$ and hydrolyses substrates such as horse serum glycoproteins [51]. The homologous enzyme from $C$. diphtheriae was characterized previously and shown to contain neuraminidase and trans-sialidase activities $[52,53]$. In principle, neuraminidases are a distinct class of glycosyl hydrolases that catalyze the removal of terminal sialic acids from various glycoconjugates and contribute to the recognition of sialic acids exposed on host cell surfaces, whereas trans-sialidases can be used for the decoration of various acceptor molecules on the cell surface to enable the invasion of host cells under certain conditions [54].
Therefore, microbial neuraminidases and trans-sialidases have the general capacity to modify the ability of host cells to respond to bacterial infections and are thus of importance for any pathogenic microorganism.

Furthermore, two gene clusters detected in each $C$. ulcerans genome are considered to encode adhesive pilus structures that are covalently anchored to the corynebacterial cell wall and probably mediate the initial adhesion of the pathogen to host tissues (Table 5). Different types of adhesive pili can presumably allow the pathogen to interact with different receptors on the host cell surface and to facilitate the delivery of virulence factors and intracellular invasion. The genetic organization of the first gene cluster is similar to the spaDEF gene region of $C$. diphtheriae NCTC 13129 encoding the SpaDEF pilus [55]. This adhesive pilus of $C$. diphtheriae is composed of the major pilin SpaD, the minor pilin subunit $\mathrm{SpaE}$ and the tip protein SpaF. The assembly of the SpaDEF precursors into a high-molecular-weight pilus structure requires the pilus-specific sortases SrtB and SrtC that are encoded within the $s p a D E F$ gene region of $C$. diphtheriae [55]. Likewise, the sortase genes $\operatorname{srtB}$ and $s r t C$ are present in the spaDEF regions of $C$. ulcerans 809 and C. ulcerans BR-AD22 and probably involved (in conjunction with the housekeeping sortase gene $\operatorname{srtD}$ [56]) in the assembly of a distinct pilus structure on the cell surface of these strains.

The second pilus gene cluster detected in the genomes of the $C$. ulcerans strains consists of the spaBC genes and the single sortase gene $s r t A$. The $s p a B$ gene encodes a minor pilus protein, whereas the $s p a C$ gene codes for a tip protein. The respective adhesive pilus of $C$. ulcerans thus lacks a major pilin subunit, unless it can be replaced by the major pilin SpaD of the SpaDEF pilus. However,

Table 5 Overview of candidate virulence factors detected in the $C$. ulcerans genomes

\begin{tabular}{lllll}
\hline ID in 809 & ID in BR-AD22 & Gene & Proposed function of deduced protein & LPXTG $^{\text {a }}$ \\
\hline CULC809_00177 & - & rbp & Ribosome-binding protein & none \\
CULC809_01974 & CULC22_02125 & cpp & Corynebacterial protease CP40 & none \\
CULC809_00040 & CULC22_00038 & pld & Phospholipase D & none \\
CULC809_01949 & CULC22_02103 & spaF & Surface-anchored protein (pilus subunit) & LPKTG \\
CULC809_01950 & CULC22_02104 & spaE & Surface-anchored protein (pilus subunit) & LPLTG \\
CULC809_01952 & CULC22_02106 & spaD & Surface-anchored protein (pilus subunit) & LPMTG \\
CULC809_01979 & CULC22_02130 & spaC & Surface-anchored protein (pilus subunit) & LPLTG \\
CULC809_01980 & CULC22_02131 & spaB & Surface-anchored protein (pilus subunit) & LARTG \\
CULC809_01133 & CULC22_01148 & rpfl & Rpf interacting protein & none \\
CULC809_01521 & CULC22_01537 & cwlH & Cell wall-associated hydrolase & none \\
CULC809_00434 & CULC22_00437 & nanH & Sialidase precursor (neuraminidase H) & none \\
CULC809_00509 & CULC22_00515 & vsp1 & Venome serine protease & none \\
CULC809_01964 & - & Venome serine protease & none \\
CULC809_01848 & CULC22_02007 & tspA & Trypsin-like serine protease & none \\
\hline
\end{tabular}

a Predicted LPXTG motif used for anchoring of the protein to the cell wall

${ }^{\mathrm{b}}$ Data presented in this manuscript indicates a function as endoglycosidase. The gene should therefore be renamed as $n d o E$. 
comprehensive studies in $C$. diphtheriae demonstrated that the adhesive pili of strain NCTC 13129 are independently assembled and are morphologically distinct $[55,57,58]$, suggesting that a replacement of the major pilin subunit is unlikely in C. ulcerans. Interestingly, the adherence of $C$. diphtheriae to human pharyngeal epithelial cells can be mediated also by the minor pilin $\mathrm{SpaB}$ of the SpaABC pilus [59]. Therefore, it is likely that homodimeric or heterodimeric $\mathrm{SpaB} / \mathrm{SpaC}$ proteins are anchored covalently to the cell surface of $C$. ulcerans 809 and $C$. ulcerans $\mathrm{BR}-\mathrm{AD} 22$ and provide tight contact between the bacterial cells and the host tissue in the absence of a pilus shaft [60].

Moreover, genes encoding homologs of the resuscitation-promoting factor interacting protein DIP1281 (RpfI/RipA) and the cell wall-associated hydrolase DIP1621 (CwlH) from C. diphtheriae NCTC 13129 were detected in the genomes of C. ulcerans 809 and $C$. ulcerans BR-AD22 (Table 5). Both conserved enzymes are involved in organizing the corynebacterial cell surface and contribute-probably indirectly-to the adhesion of $C$. diphtheriae to epithelial cells and the subsequent internalization of this pathogen [61-63].

\section{Prominent virulence factors of $C$. ulcerans: phospholipase $D$, endoglycosidase EndoE and the Shiga toxin-like ribosome-binding protein $\mathrm{Rbp}$}

A well-established virulence factor of C. ulcerans is the toxic phospholipase D (Pld) that shows amino acid sequence similarity to secreted phospholipases from $C$. pseudotuberculosis and Arcanobacterium haemolyticum [18]. While sequence homologs of the Pld enzyme are not found elsewhere in bacterial species, a similar enzyme is produced as an exotoxin in Loxosceles spiders. The spider and bacterial enzyme were shown to hydrolyze albumin-bound lysophosphatidylcholine, yielding the lipid mediator lysophosphatidic acid, a known inducer of pro-inflammatory responses [64]. The pld gene was detected in both C. ulcerans genomes, which confirms previous PCR assays performed during the initial characterization of the two isolates [15,25]. Moreover, the cpp gene (CULC809_01974 and CULC22_02125) encoding "corynebacterial protease $\mathrm{CP} 40$ " is present in the genome sequences of C. ulcerans 809 and C. ulcerans BR-AD22 (Table 5). The homologous enzyme from C. pseudotuberculosis was identified as a protective antigen against caseous lymphadenitis $[46,47]$ and shown to be of the serine protease type as the purified protein revealed proteolytic activity in a gelatine SDS-PAGE assay [65]. On the other hand, database searches did not identify any active-site homologies with other serine proteases and protease activity was undetectable in culture supernatants of C. pseudotuberculosis [65]. As presented in Figure 5A, the amino acid sequences of Cpp from C. ulcerans 809 and C. pseudotuberculosis FRC41 revealed striking similarities to the $\alpha$-domain of the extracellular endoglycosidase EndoE from Enterococcus faecalis [66]. The three proteins contain the conserved FGH18 motif assigning them to the glycosyl hydrolases of family 18 that includes enzymes with endo- $\beta-N$-acetylglucosaminidase activity [67]. EndoE from E. faecalis is a two-domain protein that is characterized by two distinct activities involved in the degradation of $N$-linked glycans from ribonuclease $B$ and the hydrolysis of the conserved glycans on IgG [66]. The latter activity of the enzyme was assigned exclusively to the $\beta$-domain of the EndoE protein, suggesting that the homologous counterparts from $C$. ulcerans have only a single activity as endoglycosidase. Nevertheless, both $C$. ulcerans strains are probably able to interact with the mammalian host by glycolytic modulation of host glycoproteins.

As described above, the group of singletons detected in the genome sequence of $C$. ulcerans 809 comprises the $r b p$ gene (CULC809_00177) that encodes a putative ribosome-binding protein containing the Pfam domain 00161 named "ribosome inactivating protein" [68]. The deduced amino acid sequence of $r b p$ shows weak similarity to the A chains of the Shiga-like toxins SLT-1 and SLT-2 from Escherichia coli (Figure 5B). The SLT-1 protein of E. coli belongs to the ribosome-binding protein type II family that is characterized by low similarity on the amino acid sequence level and, on the other hand, by a highly conserved tertiary structure of the family members [69]. The ribosome-binding protein identified in the $C$. ulcerans 809 genome shares only $24 \%$ identity with the A chain of SLT-1, but comprises all highly conserved amino acid residues needed for the catalytic $N$-glycosidase activity (Figure 5B). Moreover, an in silico comparison of the tertiary structures of Rbp and the A chain of SLT-1 demonstrates significant structural similarities of both proteins (Figure 5B). SLT-1 is usually composed of a catalytic A chain that is non-covalently associated with a pentamer of B chains [70]. The B subunits of SLT-1 are essential for binding a specific glycolipid receptor and the subsequent translocation of the toxin into the endoplasmatic reticulum (ER) of the host cell. The ER-targeting sequence of SLT-1 leads to retranslocation of the catalytic domain into the cytosol and the subsequent inhibition of protein biosynthesis by depurination of a single adenosine residue in the $28 \mathrm{~S}$ rRNA of the eukaryotic ribosome [70]. In vitro, SLT-1 has been demonstrated to induce apoptosis in endothelial cells isolated from various anatomical sites [71]. The analysis of the amino acid sequence of Rbp revealed the lack of the ER-targeting sequence at the C-terminal end of the protein from C. ulcerans 809. However, as C. ulcerans can probably persist as a facultative intracellular pathogen in mammalian host cells, a retranslocation of the toxin into the cytosol is not 
(A)

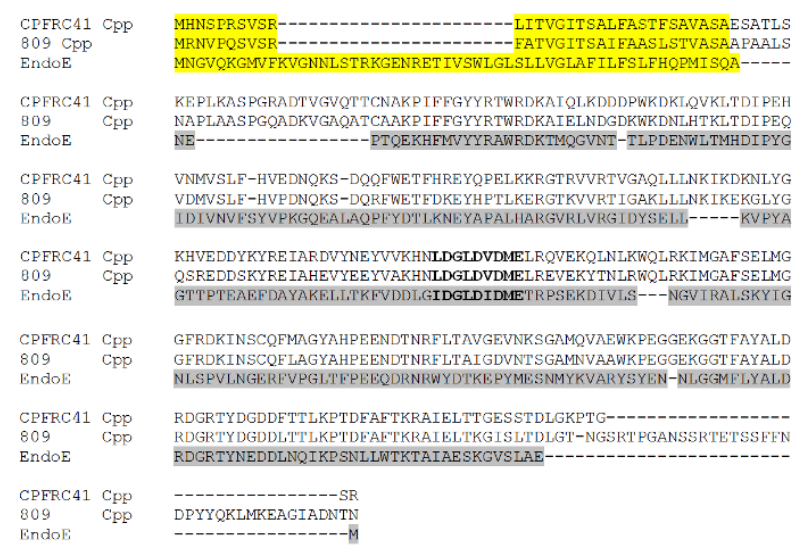

schematic map of EndoE

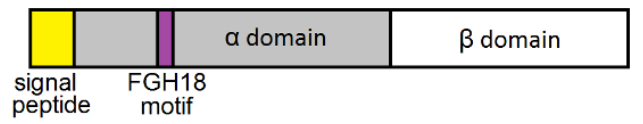

(B)

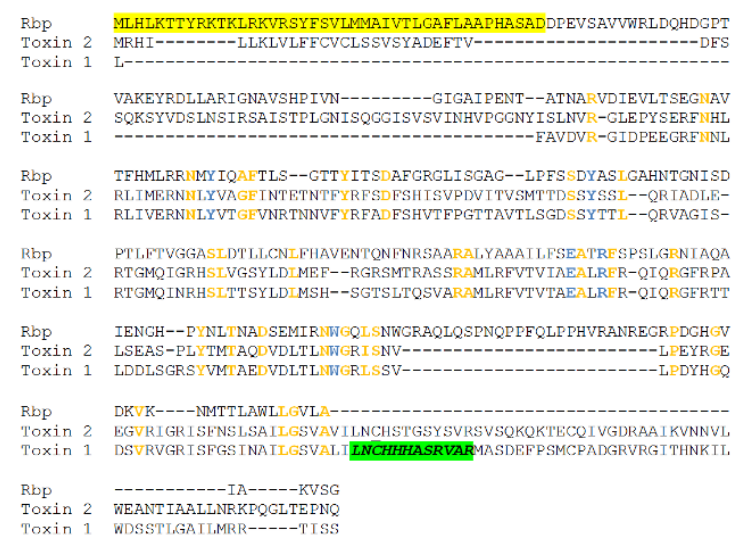

structure alignment

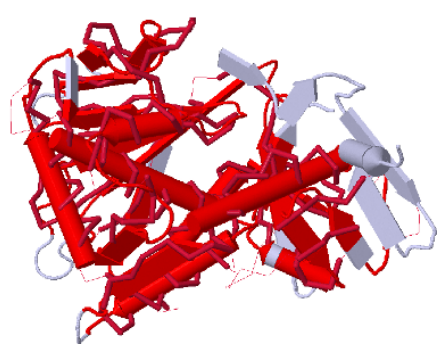

Figure 5 Sequence analyses of prominent virulence factors detected in the genome of C. ulcerans 809. (A), Analysis of the corynebacterial protease CP40 (Cpp). An amino acid sequence alignment of the corynebacterial proteases CP40 from C. pseudotuberculosis FRC41 and C. ulcerans 809 with the $\alpha$ domain of endoglycosidase EndoE from E. faecalis is shown. Predicted signal peptides are colored in yellow; predicted protein segments belonging to the $\alpha$ domain of EndoE are shaded gray. The catalytic FGH18 motif of EndoE is indicated by bold letters. The domain organization of EndoE is shown schematically below the sequence alignment. (B), Analysis of the corynebacterial ribosomebinding protein (Rbp). An amino acid sequence alignment of Rbp from C. ulcerans 809 with A chains of the Shiga-like toxins SLT-1 and SLT-2 from E. coli is shown. Conserved amino acids are highlighted in orange, while the conserved catalytic residues are highlighted in blue. The predicted signal peptide of Rbp is labeled yellow; the retranslocation domain of SLT-1 is marked as a green box. The similarity between Rbp and the A chain of SLT-1 is also shown as a 3-D model presented below the sequence alignment. Structural similarities between both proteins are indicated in red.

necessary. The secretion of the putative toxin into the cytosol of the host cell is supported instead by a typical signal sequence at the $\mathrm{N}$-terminus of the protein (Figure $5 \mathrm{~B})$. As the enzymatic activity of the ribosome-binding protein Rbp leads to inhibition of protein biosynthesis, as is the case with diphtheria toxin [72], C. ulcerans 809 could have mimicked the systemic symptoms of diphtheria in the infected patient without carrying a lysogenic $\beta$-corynephage [54].

\section{Conclusions}

The analysis of the complete genome sequences from $C$. ulcerans 809 and C. ulcerans BR-AD22 provides detailed insights into the genome architecture and the repertoire of candidate genes contributing to the virulence of these strains. Both C. ulcerans isolates differ in the number of prophage-like elements in the genome and lack sequences with similarity to the $\beta$-corynephage encoding the diphtheria toxin. Therefore, the sequenced C. ulcerans isolates can be regarded in principle as "non- toxigenic" with respect to the synthesis of diphtheria toxin. This result of the genome sequence annotation is remarkable, as previous PCR studies and toxigenicity assays with C. ulcerans 809 suggested the presence of a diphtheria-like toxin in this strain that was isolated from a patient with pulmonary infection [15]. However, the comparison of tox genes showed differences in the amino acid sequences of the diphtheria toxin from $C$. diphtheriae and C. ulcerans isolates from extrapharyngeal infections [2,3]. This data indicated that the diphtheria toxin sequences from C. ulcerans isolates are less well conserved than their counterparts from $C$. diphtheriae [3]. Moreover, the examination of diagnostic PCR assays for the detection of the tox gene in clinical samples revealed atypical results in some C. ulcerans strains [73], indicating that a reliable diagnostic is necessary for the precise characterization of C. ulcerans strains from clinical specimens [74]. The absence of the diphtheria toxin gene in C. ulcerans 809 moreover explains why the medical treatment of the elderly 
patient with a diphtheria antitoxin was unsuccessful [15].

The integration site of the $\beta$-corynephage is located at a tRNA ${ }^{\mathrm{Arg}}$ gene in the genomes of $C$. diphtheriae and $C$. ulcerans $[75,76]$. A remnant of a $\beta$-like phage was detected at this genomic position in the genome sequence of C. ulcerans 809 , and the $r b p$ gene encoding a ribosome-binding protein is present in the immediate vicinity. The low $\mathrm{G}+\mathrm{C}$ content of the $r b p$ gene is suggestive of the horizontal transfer of this coding region to $C$. ulcerans 809 . Whether the $r b p$ gene was a former part of a corynephage or associated with an adjacent transposable element cannot be deduced from the current data and remains to be elucidated. The Rbp protein of $C$. ulcerans 809 revealed structural similarity to the A chain of the Shiga-like toxin SLT-1 from E. coli [69]. The SLT-1 protein has been implicated in the pathogenesis of acute renal failure [71] that was also diagnosed in the elderly Brazilian woman with pulmonary infection [15]. Therefore, the Rbp protein of C. ulcerans 809 may represent a prominent virulence factor by inhibiting the protein biosynthesis in host cells due to the putative ribosome-binding activity.

Interestingly, the $r b p$ gene is absent in the genome sequence of $C$. ulcerans BR-AD22 that was isolated from an asymptomatic dog [25]. As the current knowledge of $C$. ulcerans is biased due to the predominant recovery of toxigenic strains from respiratory diphtheria-like illnesses in humans, future work should include strains from extrapharyngeal specimens and particularly from various animal sources. The sequencing of more C. ulcerans genomes from different habitats will open the way to the pan-genomic level of comparative genomics. A comparative approach with a larger set of sequenced $C$. ulcerans genomes may help to gain insights into the distinctive features of strains from human and animal sources and to describe the proposed zoonotic transmission of this pathogen in more detail.

It is moreover necessary to clarify the physiological role of the predicted "corynebacterial protease CP40". In a previous study, the homologous enzyme from C. pseudotuberculosis was assigned to the serine protease family due to its associated proteolytic activity although no protease activity could be detected in C. pseudotuberculosis culture supernatants [65]. In contrast to this data, the in silico analysis of the domain organization of CP40 in this study and previous phylogenetic data of glycosyl hydrolases strongly indicate that this protein is an endoglycosidase with similarity to the $\alpha$ domain of EndoE from E. faecalis [66]. The $\alpha$ domain of EndoE hydrolyzes the glycans on RNase B, which could be important for the pathogenesis and persistence of a bacterium during human infections [66]. We therefore suggest renaming the corresponding genes from $C$. ulcerans and $C$.

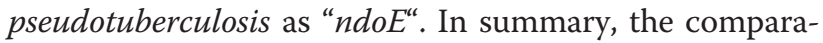
tive analysis of two complete C. ulcerans genomes provides new valuable information on known virulence factors and detected novel candidate genes probably contributing to the virulence of this species. According to the genome annotation, the repertoire of prominent virulence factors from $C$. ulcerans 809 comprises the phospholipase D, the neuraminidase NanH, the novel ribosome-binding protein $\mathrm{Rbp}$, and the endoglycosidase EndoE (formerly named corynebacterial protease CP40).

\section{Methods}

\section{Bacterial strains and growth conditions}

C. ulcerans 809 was originally isolated in Rio de Janeiro from a bronchoalveolar lavage sample of an elderly woman with a fatal pulmonary infection and a history of leg skin ulcers [15]. C. ulcerans BR-AD22 was previously obtained from a nasal sample of an asymptomatic female dog kept in an animal shelter in the metropolitan area of Rio de Janeiro [25]. Both isolates were assigned to the species C. ulcerans by taxonomic assays and sequencing of the 16S rDNA. The clinical history of the patient and the examination of the respective dog were described in detail in previous studies [15,25]. Both $C$. ulcerans strains were routinely grown at $37^{\circ} \mathrm{C}$ in brainheart-infusion (BHI) broth or on Columbia agar supplemented with $5 \%$ sheep blood.

Preparation of chromosomal DNA for genome sequencing The preparation of chromosomal DNA from C. ulcerans 809 and C. ulcerans BR-AD22 was performed as described previously [21]. Briefly, 50-ml aliquots of bacterial cultures grown for 48-72 $\mathrm{h}$ in $\mathrm{BHI}$ broth were centrifuged at $4^{\circ} \mathrm{C}$ and $2,000 \times g$ for $20 \mathrm{~min}$. The resulting cell pellets were resuspended in $0.6 \mathrm{ml}$ Tris $/ \mathrm{NaCl}$ buffer [10 mM Tris (pH 7.0), 10 mM EDTA, $300 \mathrm{mM}$ $\mathrm{NaCl}$ ] and transferred to VK01 Precellys lysing tubes. The bacterial cells were lysed by means of a Precellys 24-Dual Tissue Homogenizer using two cycles of 6,500 rpm for $15 \mathrm{sec}$ with an interval of $30 \mathrm{sec}$. The chromosomal DNA was subsequently purified by extraction with phenol/chloroform/isoamyl alcohol (25:24:1) and precipitated with ethanol. DNA concentrations were determined with a Tecan Infinite 200 Microplate Reader.

\section{Genome sequencing of C. ulcerans 809 and C. ulcerans BR-AD22}

Single-stranded template DNA libraries for genome sequencing of C. ulcerans 809 und C. ulcerans BR-AD22 were established by using $5 \mu \mathrm{g}$ of purified chromosomal DNA for each strain. The preparation of the DNA libraries was carried out according to standard protocols from Roche Applied Science. The DNA concentration 
of the resulting libraries was measured with the Agilent RNA 6000 Nano Kit. DNA sequencing was performed with the Genome Sequencer FLX Instrument and Titanium chemistry (Roche Applied Science). The genomic sequences were assembled with the Newbler Assembler software (version 2.3) and the results were documented in the respective 454 Newbler Metrics files. The automatic assembly of genomic sequences from C. ulcerans 809 yielded 14 large (> 500 bases) and 8 small contigs, together composed of 250,786 assembled reads representing 106,993,163 sequenced bases. The assembly of genomic sequences from C. ulcerans BR-AD22 led to 25 large and 3 small contigs, based on 178,615 assembled reads representing the total number of $59,757,327$ sequenced bases. The subsequent gap closure process was facilitated by in silico predictions of the contig order that were computed by the related reference contig arrangement tool r2cat using the C. pseudotuberculosis FRC41 genome sequence as a reference and the default parameters of the integrated $q$-gram filter $[21,26]$. All matching regions were displayed in an interactive synteny plot and oriented automatically according to their matches, using a sliding window approach that determines the position of a contig on the reference genome [26]. The remaining gaps in the genome sequences were closed by PCR assays with Phusion hot start high-fidelity DNA polymerase (Finnzymes) and genomic template DNAs. The PCR assays were performed according to standard protocols from Finnzymes using $1 \mathrm{M}$ betain for efficient denaturation of DNA secondary structures. The amplified DNA fragments linking individual contigs were sequenced by IIT Biotech (Bielefeld, Germany). All contigs and additional DNA sequences were uploaded into the Consed program [77] to finish the genome sequences of C. ulcerans 809 and C. ulcerans BR-AD22.

\section{Annotation and bioinformatic analysis of the genome sequences}

The annotations of the assembled genome sequences of C. ulcerans 809 and C. ulcerans BR-AD22 were performed with the GenDB system that supports automatic annotation strategies and manual data curation [27]. In the GenDB annotation system, a combined gene prediction strategy was executed by means of REGANOR, GLIMMER 2.1 and the CRITICA program suite along with postprocessing by the RBSfinder tool. The predicted proteins of C. ulcerans were functionally characterized by automated searches in public databases, including SWISS-PROT, TrEMBL, Pfam, TIGRFAM, KEGG, COG, CDD and Interpro. Finally, an automated functional annotation was performed using default parameters. The origin of chromosomal DNA replication was predicted with the Ori-Finder program [28];
CRISPRs were detected with the CRISPRFinder tool [36]. Both tools were applied with their recent web versions using default parameters. Metabolic properties of the sequenced $C$. ulcerans strains were deduced from in silico reconstructions of metabolic networks with the software CARMEN using metabolic pathway information from the KEGG database and from manually curated SBML templates [78]. The predicted C. ulcerans proteins were mapped onto the SBML templates by means of bidirectional best BLASTP hits using the scoring matrix BLOSUM62 and the user-defined E-value cutoff of $1 \times 10^{-10}$. The synteny between corynebacterial genomes was calculated by the EDGAR software with default parameters [33]. Comparative analysis of the predicted gene content of C. ulcerans 809 and C. ulcerans BR-AD22 was also performed with the EDGAR platform. This analysis was based on the calculation of BLAST score ratio values using the pre-calculated corynebacterial master cutoff of 77 [33]. Secreted proteins were detected with SignalP 3.0 using the default settings for gram-positive bacteria [48]. Multiple amino acid sequence alignments were generated with the Clustal W 1.82 program [79]. The comparison of tertiary structures of proteins was computed by means of the Dali server using default parameters. This server computes structural alignments between two protein structures using the DaliLite-pairwise option (version 3.1) [80]. The PDB file for Rbp was calculated with the SWISS-Model workspace automated modeling mode using the SLT-1 A1 chain (PDB code $1 \mathrm{dm} 0$ ) as reference input [81]. The genome sequences of C. ulcerans 809 (CP002790) and C. ulcerans BR-AD22 (CP002791) have been deposited in the GenBank database.

\section{Acknowledgements}

ET and JS acknowledge the receipt of scholarships that are embedded into the Cluster Industrial Biotechnology CLIB ${ }^{2021}$ and financed by Bielefeld University and the Ministry of Innovation, Science, Research and Technology of North Rhine-Westphalia. AB was supported by the Deutsche Forschungsgemeinschaft embedded in SFB 796 (project B5). The authors thank Jochen Blom, Sebastian Jaenicke and Burkhard Linke for bioinformatics support. The valuable contribution of the CeBiTec sequencing team (A. Albersmeier, K. Brinkrolf, D. K. Cavalcanti de Lucena, S. Schatschneider, R. Szczepanowski, A. Winkler) to this genome project is greatly acknowledged. We acknowledge support of the publication fee by Deutsche Forschungsgemeinschaft and the Open Access Publication Funds of Bielefeld University.

\section{Author details}

'Institut für Genomforschung und Systembiologie, Centrum für Biotechnologie, Universität Bielefeld, Universitätsstraße 27, D-33615 Bielefeld, Germany. ${ }^{2}$ CLIB Graduate Cluster Industrial Biotechnology, Centrum für Biotechnologie, Universität Bielefeld, Universitätsstraße 27, D-33615 Bielefeld, Germany. ${ }^{3}$ Bioinformatics Resource Facility, Centrum für Biotechnologie, Universität Bielefeld, Universitätsstraße 25, D-33615 Bielefeld, Germany. ${ }^{4}$ Lehrstuhl für Genomforschung, Fakultät für Biologie, Universität Bielefeld, Universitätsstraße 27, D-33615 Bielefeld Germany. ${ }^{5}$ Lehrstuhl für Mikrobiologie, Friedrich-Alexander-Universität Erlangen-Nürnberg, Staudtstraße 5, D-91058 Erlangen, Germany. 'Laboratório de Genética Celular e Molecular, Departamento de Biologia Geral, Instituto de Ciências 
Biológicas, Universidade Federal de Minas Gerais, Av. Antonio Carlos 6627, Pampulha, Belo Horizonte, MG, Brazil. ${ }^{7}$ Instituto de Ciências Biológicas, Universidade Federal do Pará, Rua Augusto Corrêa, 01-Guamá, Belém, PA, Brazil. ${ }^{8}$ Faculdade de Ciências Médicas, Universidade do Estado do Rio de Janeiro, Av. 28 de Setembro 87, 20551-030 Rio de Janeiro, RJ, Brazil.

\section{Authors' contributions}

ET sequenced and annotated the C. ulcerans genomes and prepared the manuscript. AA and PP participated in genome annotation with GenDB. JS implemented the GenDB project and provided bioinformatic support. PV participated in the gap closure process. SS, SA and FD purified the genomic DNAs. The comparative genome analysis was supported by $A B, A M, V A, M S$ and $A S . C S, L S, P S, A D, R H$ and $A M$ isolated and characterized the C. ulcerans strains prior to this study and participated in the comparative analysis. AT coordinated and supervised the project and the comparative analysis. All authors read and approved the final version of the manuscript

Received: 1 June 2011 Accepted: 30 July 2011 Published: 30 July 2011

\section{References}

1. Gilbert R, Stewart FC: Corynebacterium ulcerans: a pathogenic microorganism resembling C. diphtheriae. J Lab Clin Med 1926, 12:756-761.

2. $\quad$ Sing $A$, Hogardt M, Bierschenk $S$, Heesemann J: Detection of differences in the nucleotide and amino acid sequences of diphtheria toxin from Corynebacterium diphtheriae and Corynebacterium ulcerans causing extrapharyngeal infections. J Clin Microbiol 2003, 41:4848-4851.

3. Sing A, Bierschenk S, Heesemann J: Classical diphtheria caused by Corynebacterium ulcerans in Germany: amino acid sequence differences between diphtheria toxins from Corynebacterium diphtheriae and $C$. ulcerans. Clin Infect Dis 2005, 40:325-326.

4. Hadfield TL, McEvoy P, Polotsky Y, Tzinserling VA, Yakovlev AA: The pathology of diphtheria. J Infect Dis 2000, 181(Suppl 1):S116-120.

5. Tiwari TS, Golaz A, Yu DT, Ehresmann KR, Jones TF, Hill HE, Cassiday PK, Pawloski LC, Moran JS, Popovic T, Wharton M: Investigations of 2 cases of diphtheria-like illness due to toxigenic Corynebacterium ulcerans. Clin Infect Dis 2008, 46:395-401.

6. Wagner KS, White JM, Crowcroft NS, De Martin S, Mann G, Efstratiou A: Diphtheria in the United Kingdom, 1986-2008: the increasing role of Corynebacterium ulcerans. Epidemiol Infect 2010, 138:1519-1530.

7. Bostock AD, Gilbert FR, Lewis D, Smith DC: Corynebacterium ulcerans infection associated with untreated milk. J Infect 1984, 9:286-288.

8. Hart RJ: Corynebacterium ulcerans in humans and cattle in North Devon. J Hyg (Lond) 1984, 92:161-164.

9. Lartigue MF, Monnet X, Le Fleche A, Grimont PA, Benet JJ, Durrbach A, Fabre M, Nordmann P: Corynebacterium ulcerans in an immunocompromised patient with diphtheria and her dog. J Clin Microbiol 2005, 43:999-1001.

10. Hogg RA, Wessels J, Hart J, Efstratiou A, De Zoysa A, Mann G, Allen T, Pritchard GC: Possible zoonotic transmission of toxigenic Corynebacterium ulcerans from companion animals in a human case of fatal diphtheria. Vet Rec 2009, 165:691-692.

11. Schuhegger R, Schoerner C, Dlugaiczyk J, Lichtenfeld I, Trouillier A, ZellerPeronnet V, Busch U, Berger A, Kugler R, Hormansdorfer S, Sing A: Pigs as source for toxigenic Corynebacterium ulcerans. Emerg Infect Dis 2009 15:1314-1315.

12. De Zoysa A, Hawkey PM, Engler K, George R, Mann G, Reilly W, Taylor D, Efstratiou A: Characterization of toxigenic Corynebacterium ulcerans strains isolated from humans and domestic cats in the United Kingdom. J Clin Microbiol 2005, 43:4377-4381.

13. Dessau RB, Brandt-Christensen M, Jensen OJ, Tonnesen P: Pulmonary nodules due to Corynebacterium ulcerans. Eur Respir J 1995, 8:651-653.

14. Nureki S, Miyazaki E, Matsuno O, Takenaka R, Ando M, Kumamoto T, Nakano T, Ohkusu K, Ezaki T: Corynebacterium ulcerans infection of the lung mimicking the histology of Churg-Strauss syndrome. Chest 2007, 131:1237-1239.

15. Mattos-Guaraldi AL, Sampaio $J$, Santos CS, Pimenta FP, Pereira GA, Pacheco LG, Miyoshi A, Azevedo V, Moreira LO, Gutierrez FL, et al: First detection of Corynebacterium ulcerans producing a diphtheria-like toxin in a case of human with pulmonary infection in the Rio de Janeiro metropolitan area, Brazil. Mem Inst Oswaldo Cruz 2008, 103:396-400.
16. Pallen MJ, Hay AJ, Puckey LH, Efstratiou A: Polymerase chain reaction for screening clinical isolates of corynebacteria for the production of diphtheria toxin. J Clin Pathol 1994, 47:353-356.

17. Dewinter LM, Bernard KA, Romney MG: Human clinical isolates of Corynebacterium diphtheriae and Corynebacterium ulcerans collected in Canada from 1999 to 2003 but not fitting reporting criteria for cases of diphtheria. J Clin Microbiol 2005, 43:3447-3449.

18. McNamara PJ, Cuevas WA, Songer JG: Toxic phospholipases D of Corynebacterium pseudotuberculosis, C. ulcerans and Arcanobacterium haemolyticum: cloning and sequence homology. Gene 1995, 156:113-118.

19. Riegel P, Ruimy R, de Briel D, Prevost G, Jehl F, Christen R, Monteil H: Taxonomy of Corynebacterium diphtheriae and related taxa, with recognition of Corynebacterium ulcerans. sp nov nom rev FEMS Microbiol Lett 1995, 126:271-276.

20. Funke G, von Graevenitz A, Clarridge JE, Bernard KA: Clinical microbiology of coryneform bacteria. Clin Microbiol Rev 1997, 10:125-159.

21. Trost E, Ott L, Schneider J, Schroder J, Jaenicke S, Goesmann A, Husemann P, Stoye J, Dorella FA, Rocha FS, et al: The complete genome sequence of Corynebacterium pseudotuberculosis FRC41 isolated from a 12-year-old girl with necrotizing lymphadenitis reveals insights into gene-regulatory networks contributing to virulence. BMC Genomics 2010, 11:728.

22. Silva A, Schneider MP, Cerdeira L, Barbosa MS, Ramos RT, Carneiro AR, Santos R, Lima M, D'Afonseca V, Almeida SS, et al: Complete genome sequence of Corynebacterium pseudotuberculosis 119, a strain isolated from a cow in Israel with bovine mastitis. J Bacteriol 2010, 193:323-324

23. Ruiz JC, D'Afonseca V, Silva A, Ali A, Pinto AC, Santos AR, Rocha AA, Lopes DO, Dorella FA, Pacheco LG, et al: Evidence for reductive genome evolution and lateral acquisition of virulence functions in two Corynebacterium pseudotuberculosis strains. PLoS One 2011, 6:e18551.

24. Cerdeno-Tarraga AM, Efstratiou A, Dover LG, Holden MT, Pallen M, Bentley SD, Besra GS, Churcher C, James KD, De Zoysa A, et al: The complete genome sequence and analysis of Corynebacterium diphtheriae NCTC13129. Nucleic Acids Res 2003, 31:6516-6523.

25. Dias AA, Silva FC Jr, Pereira GA, Souza MC, Camello TC, Damasceno JA, Pacheco LG, Miyoshi A, Azevedo VA, Hirata R Jr, et al: Corynebacterium ulcerans isolated from an asymptomatic dog kept in an animal shelter in the metropolitan area of Rio de Janeiro, Brazil. Vector Borne Zoonotic Dis 2010, 10:743-748.

26. Husemann P, Stoye J: r2cat: synteny plots and comparative assembly. Bioinformatics 2010, 26:570-571.

27. Meyer F, Goesmann A, MCHardy AC, Bartels D, Bekel T, Clausen J, Kalinowski J, Linke B, Rupp O, Giegerich R, Pühler A: GenDB-an open source genome annotation system for prokaryote genomes. Nucleic Acids Res 2003, 31:2187-2195.

28. Gao F, Zhang CT: Ori-Finder: a web-based system for finding oriCs in unannotated bacterial genomes. BMC Bioinformatics 2008, 9:79.

29. Hendrickson H, Lawrence JG: Mutational bias suggests that replication termination occurs near the dif site, not at Ter sites. Mol Microbiol 2007, 64:42-56.

30. Hendrickson H, Lawrence JG: Selection for chromosome architecture in bacteria. J Mol Evol 2006, 62:615-629.

31. Tauch A, Trost E, Tilker A, Ludewig U, Schneiker S, Goesmann A, Arnold W, Bekel T, Brinkrolf $K$, Brune I, et al: The lifestyle of Corynebacterium urealyticum derived from its complete genome sequence established by pyrosequencing. J Biotechnol 2008, 136:11-21.

32. Trost E, Götker S, Schneider J, Schneiker-Bekel S, Szczepanowski R, Tilker A, Viehoever P, Arnold W, Bekel T, Blom J, et al: Complete genome sequence and lifestyle of black-pigmented Corynebacterium aurimucosum ATCC 700975 (formerly C. nigricans CN-1) isolated from a vaginal swab of a woman with spontaneous abortion. BMC Genomics 2010, 11:91.

33. Blom J, Albaum SP, Doppmeier D, Pühler A, Vorhölter FJ, Zakrzewski M, Goesmann A: EDGAR: a software framework for the comparative analysis of prokaryotic genomes. BMC Bioinformatics 2009, 10:154.

34. Buck GA, Cross RE, Wong TP, Loera J, Groman N: DNA relationships among some tox-bearing corynebacteriophages. Infect Immun 1985, 49:679-684.

35. Rappuoli R, Michel JL, Murphy JR: Integration of corynebacteriophages beta tox ${ }^{+}$, omega tox ${ }^{+}$, and gamma tox into two attachment sites on the Corynebacterium diphtheriae chromosome. J Bacteriol 1983, 153:1202-1210. 
36. Grissa I, Vergnaud G, Pourcel C: CRISPRFinder: a web tool to identify clustered regularly interspaced short palindromic repeats. Nucleic Acids Res 2007, 35:W52-57.

37. Deveau H, Garneau JE, Moineau S: CRISPR/Cas system and its role in phage-bacteria interactions. Annu Rev Microbiol 2010, 64:475-493.

38. Mokrousov I, Vyazovaya A, Kolodkina V, Limeschenko E, Titov L, Narvskaya O: Novel macroarray-based method of Corynebacterium diphtheriae genotyping: evaluation in a field study in Belarus. Eur $J$ Clin Microbiol Infect Dis 2009, 28:701-703.

39. Ton-That $H$, Marraffini LA, Schneewind O: Protein sorting to the cell wall envelope of Gram-positive bacteria. Biochim Biophys Acta 2004, 1694:269-278

40. Yukawa H, Omumasaba CA, Nonaka H, Kos P, Okai N, Suzuki N, Suda M, Tsuge $Y$, Watanabe J, Ikeda $Y$, et al: Comparative analysis of the Corynebacterium glutamicum group and complete genome sequence of strain R. Microbiology 2007, 153:1042-1058.

41. Schröder J, Tauch A: Transcriptional regulation of gene expression in Corynebacterium glutamicum: the role of global, master and local regulators in the modular and hierarchical gene regulatory network. FEMS Microbiol Rev 2010, 34:685-737.

42. Yukawa $H$, Inui $M$, Vertès AA: Amino Acid Biosynthesis-Pathways, Regulation and Metabolic Engineering. In Genomes and Genome-Level Engineering of Amino Acid-Producing Bacteria. Edited by: Wendisch VF. Springer; 2007:349-401

43. Barksdale L, Linder R, Sulea IT, Pollice M: Phospholipase D activity of Corynebacterium pseudotuberculosis (Corynebacterium ovis) and Corynebacterium ulcerans, a distinctive marker within the genus Corynebacterium. J Clin Microbiol 1981, 13:335-343.

44. McKean S, Davies J, Moore R: Identification of macrophage induced genes of Corynebacterium pseudotuberculosis by differential fluorescence induction. Microbes Infect 2005, 7:1352-1363.

45. McKean SC, Davies JK, Moore RJ: Expression of phospholipase D, the major virulence factor of Corynebacterium pseudotuberculosis, is regulated by multiple environmental factors and plays a role in macrophage death. Microbiology 2007, 153:2203-2211.

46. Walker J, Jackson HJ, Eggleton DG, Meeusen EN, Wilson MJ, Brandon MR: Identification of a novel antigen from Corynebacterium pseudotuberculosis that protects sheep against caseous lymphadenitis. Infect Immun 1994, 62:2562-2567.

47. Dorella FA, Pacheco LG, Seyffert N, Portela RW, Meyer R, Miyoshi A Azevedo V: Antigens of Corynebacterium pseudotuberculosis and prospects for vaccine development. Expert Rev Vaccines 2009, 8:205-213.

48. Emanuelsson O, Brunak S, von Heijne G, Nielsen H: Locating proteins in the cell using TargetP, SignalP and related tools. Nat Protoc 2007, 2:953-971.

49. Marraffini LA, Dedent AC, Schneewind O: Sortases and the art of anchoring proteins to the envelopes of gram-positive bacteria. Microbiol Mol Biol Rev 2006, 70:192-221.

50. Dubin G: Extracellular proteases of Staphylococcus spp. Biol Chem 2002, 383:1075-1086

51. Vertiev YV, Ezepchuk YV: Purification and characterization of some enzymatic properties of neuraminidase from Corynebacterium ulcerans. Hoppe Seylers Z Physiol Chem 1981, 362:1339-1344.

52. Kim S, Oh DB, Kwon O, Kang HA: Identification and functional characterization of the $\mathrm{NanH}$ extracellular sialidase from Corynebacterium diphtheriae. J Biochem 2010, 147:523-533.

53. Mattos-Guaraldi AL, Formiga LC, Andrade AF: Trans-sialidase activity for sialic acid incorporation on Corynebacterium diphtheriae. FEMS Microbiol Lett 1998, 168:167-172.

54. Mattos-Guaraldi AL, Duarte Formiga LC, Pereira GA: Cell surface components and adhesion in Corynebacterium diphtheriae. Microbes Infect 2000, 2:1507-1512.

55. Gaspar AH, Ton-That $\mathrm{H}$ : Assembly of distinct pilus structures on the surface of Corynebacterium diphtheriae. J Bacteriol 2006, 188:1526-1533.

56. Swaminathan A, Mandlik A, Swierczynski A, Gaspar A, Das A, Ton-That H: Housekeeping sortase facilitates the cell wall anchoring of pilus polymers in Corynebacterium diphtheriae. Mol Microbiol 2007, 66:961-974.

57. Ton-That H, Schneewind O: Assembly of pili on the surface of Corynebacterium diphtheriae. Mol Microbiol 2003, 50:1429-1438.
58. Swierczynski A, Ton-That H: Type III pilus of corynebacteria: Pilus length is determined by the level of its major pilin subunit. J Bacterio/ 2006, 188:6318-6325

59. Mandlik A, Swierczynski A, Das A, Ton-That H: Corynebacterium diphtheriae employs specific minor pilins to target human pharyngeal epithelial cells. Mol Microbiol 2007, 64:111-124.

60. Chang C, Mandlik A, Das A, Ton-That H: Cell surface display of minor pilin adhesins in the form of a simple heterodimeric assembly in Corynebacterium diphtheriae. Mol Microbiol 79:1236-1247.

61. Hett EC, Chao MC, Deng LL, Rubin EJ: A mycobacterial enzyme essential for cell division synergizes with resuscitation-promoting factor. PLOS Pathog 2008, 4:e1000001.

62. Ott L, Holler M, Gerlach RG, Hensel M, Rheinlaender J, Schaffer TE, Burkovski A: Corynebacterium diphtheriae invasion-associated protein (DIP1281) is involved in cell surface organization, adhesion and internalization in epithelial cells. BMC Microbio/ 2010, 10:2

63. Kolodkina V, Denisevich T, Titov L: Identification of Corynebacterium diphtheriae gene involved in adherence to epithelial cells. Infect Genet Evol 2011, 11:518-521.

64. van Meeteren LA, Frederiks F, Giepmans BN, Pedrosa MF, Billington SJ, Jost BH, Tambourgi DV, Moolenaar WH: Spider and bacterial sphingomyelinases D target cellular lysophosphatidic acid receptors by hydrolyzing lysophosphatidylcholine. J Biol Chem 2004, 279:10833-10836.

65. Wilson MJ, Brandon MR, Walker J: Molecular and biochemical characterization of a protective 40-kilodalton antigen from Corynebacterium pseudotuberculosis. Infect Immun 1995, 63:206-211.

66. Collin M, Fischetti VA: A novel secreted endoglycosidase from Enterococcus faecalis with activity on human immunoglobulin $\mathrm{G}$ and ribonuclease B. J Biol Chem 2004, 279:22558-22570.

67. Terwisscha van Scheltinga AC, Hennig M, Dijkstra BW: The 1.8 A resolution structure of hevamine, a plant chitinase/lysozyme, and analysis of the conserved sequence and structure motifs of glycosyl hydrolase family 18. J Mol Biol 1996, 262:243-257.

68. Marchler-Bauer A, Lu S, Anderson JB, Chitsaz F, Derbyshire MK, DeWeeseScott C, Fong JH, Geer LY, Geer RC, Gonzales NR, et al: CDD: a Conserved Domain Database for the functional annotation of proteins. Nucleic Acids Res 2011, 39:D225-229.

69. LaPointe $P$, Wei $X$, Gariepy J: A role for the protease-sensitive loop region of Shiga-like toxin 1 in the retrotranslocation of its $A 1$ domain from the endoplasmic reticulum lumen. J Biol Chem 2005, 280:23310-23318.

70. O'Loughlin EV, Robins-Browne RM: Effect of Shiga toxin and Shiga-like toxins on eukaryotic cells. Microbes Infect 2001, 3:493-507.

71. Erwert RD, Winn RK, Harlan JM, Bannerman DD: Shiga-like toxin inhibition of FLICE-like inhibitory protein expression sensitizes endothelial cells to bacterial lipopolysaccharide-induced apoptosis. J Biol Chem 2002, 277:40567-40574.

72. Holmes RK: Biology and molecular epidemiology of diphtheria toxin and the tox gene. J Infect Dis 2000, 181(Suppl 1):S156-167.

73. Cassiday PK, Pawloski LC, Tiwari T, Sanden GN, Wilkins PP: Analysis of toxigenic Corynebacterium ulcerans strains revealing potential for falsenegative real-time PCR results. J Clin Microbiol 2008, 46:331-333.

74. Schuhegger R, Lindermayer M, Kugler R, Heesemann J, Busch U, Sing A Detection of toxigenic Corynebacterium diphtheriae and Corynebacterium ulcerans strains by a novel real-time PCR. J Clin Microbiol 2008, 46:2822-2823.

75. Cianciotto N, Serwold-Davis T, Groman N, Ratti G, Rappuoli R: DNA sequence homology between attB-related sites of Corynebacterium diphtheriae, Corynebacterium ulcerans, Corynebacterium glutamicum, and the attP site of gamma-corynephage. FEMS Microbiol Lett 1990, 54:299-301.

76. Ratti G, Covacci A, Rappuoli R: A tRNA(2Arg) gene of Corynebacterium diphtheriae is the chromosomal integration site for toxinogenic bacteriophages. Mol Microbiol 1997, 25:1179-1181.

77. Gordon D, Abajian C, Green P: Consed: a graphical tool for sequence finishing. Genome Res 1998, 8:195-202.

78. Schneider J, Vorhölter FJ, Trost E, Blom J, Musa YR, Neuweger H, Niehaus K, Schatschneider S, Tauch A, Goesmann A: CARMEN-Comparative Analysis and in silico Reconstruction of organism-specific MEtabolic Networks. Genet Mol Res 2010, 9:1660-1672. 
79. Larkin MA, Blackshields G, Brown NP, Chenna R, McGettigan PA, McWilliam H, Valentin F, Wallace IM, Wilm A, Lopez R, et al: Clustal W and Clustal X version 2.0. Bioinformatics 2007, 23:2947-2948.

80. Holm L, Rosenstrom P: Dali server: conservation mapping in 3D. Nucleic Acids Res 2010, 38:W545-549.

81. Arnold K, Bordoli L, Kopp J, Schwede T: The SWISS-MODEL workspace: a web-based environment for protein structure homology modelling. Bioinformatics 2006, 22:195-201.

doi:10.1186/1471-2164-12-383

Cite this article as: Trost et al:: Comparative analysis of two complete Corynebacterium ulcerans genomes and detection of candidate virulence factors. BMC Genomics 2011 12:383.

Submit your next manuscript to BioMed Central and take full advantage of:

- Convenient online submission

- Thorough peer review

- No space constraints or color figure charges

- Immediate publication on acceptance

- Inclusion in PubMed, CAS, Scopus and Google Scholar

- Research which is freely available for redistribution

Submit your manuscript at www.biomedcentral.com/submit 